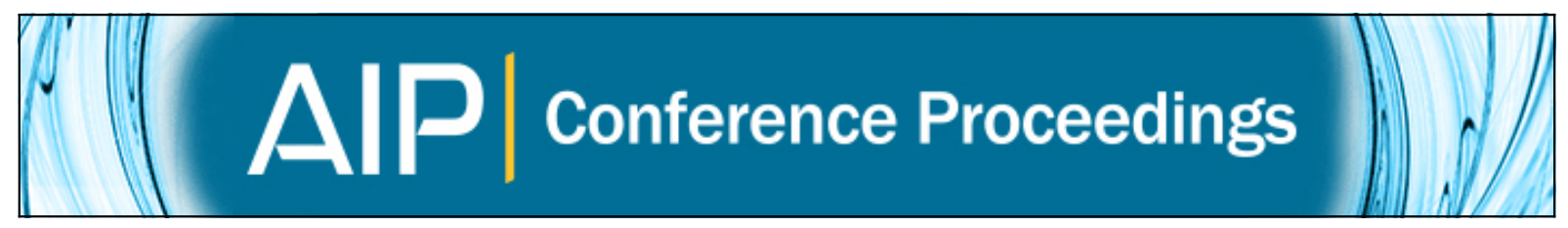

\title{
Mathematical models and numerical methods in life sciences
}

Maíra Aguiar, Roberto Cavoretto, Nico Stollenwerk, and Ezio Venturino

Citation: AIP Conference Proceedings 1738, 390001 (2016); doi: 10.1063/1.4952175

View online: http://dx.doi.org/10.1063/1.4952175

View Table of Contents: http://scitation.aip.org/content/aip/proceeding/aipcp/1738?ver=pdfcov

Published by the AIP Publishing

Articles you may be interested in

(I.) applications of mathematical methods and models in sciences and engineering AIP Conf. Proc. 1738, 290001 (2016); 10.1063/1.4952073

Commentary: New mathematical physics needed for life sciences

Phys. Today 69, 10 (2016); 10.1063/PT.3.3036

Mathematical Analysis and Numerical Methods for Science and Technology

Phys. Today 44, 72 (1991); 10.1063/1.2810363

Mathematical Methods in the Physical Sciences

Am. J. Phys. 36, 644 (1968); 10.1119/1.1975067

Mathematical Methods for Science Students

Am. J. Phys. 31, 67 (1963); 10.1119/1.1969249 


\title{
Mathematical Models and Numerical Methods in Life Sciences
}

\author{
Maíra Aguiar*, Roberto Cavoretto ${ }^{\dagger}$, Nico Stollenwerk* and Ezio Venturino $^{\dagger}$ \\ ${ }^{*}$ CMAF, Universidade de Lisboa, \\ Av. Prof. Gama Pinto 2, 1649-003 Lisboa, Portugal \\ †Dipartimento di Matematica “Giuseppe Peano", Università di Torino, \\ Via Carlo Alberto 10, 10123 Torino, Italia
}

Keywords: population dynamics, epidemiology, numerical simulations, nonlinear models, evolution, complex systems, infectious diseases, predator-prey, ecology, spatiotemporal patterns

PACS: 87.23.Cc, 87.19.Xx, 87.23.-n

After a pause, our Biomathematics session is again hosted at the International Conference of Numerical Analysis and Applied Mathematics 2015 (ICNAAM 2015), in Rhodes, Greece. Our session will present a set of contributions addressing relevant aspects on mathematical biology.

The session "Mathematical Models and Numerical Methods in Life Sciences" has both theoretical and practical applications and will cover research topics in conceptual mathematical modeling in population dynamics and epidemiology, numerical simulations of nonlinear models for understanding the evolution of complex systems involving (several) populations and/or diseases, models for the impact of infectious diseases on interacting populations. Also the important phenomenon of invasion of an environment by an alien population and the mechanisms that underlie it will be emphasized. Further, spatiotemporal patterns in ecological settings whose formation may be described both with deterministic and stochastic tools.

In population theory three papers deal with models with time lags, density-dependent selection and the phenomena of multistability that arise from them, in the case of purely demographic situations.

Ecoepidemiology appears with a contribution studying a more complex model, in that it accounts also for recovered individuals, in addition to the possibility of overcoming the disease.

Two papers deal with the problem of biodegradation of pollutants in waterbodies, obtained via the use of bacteria or aquatic plants. Also, an Inter-Algal competition via a population dynamics model with pack behaviour will be presented where a space-implicit model of ordinary differential equations (ODE) describing the evolution of two population biomass will be analyzed, showing different recruitment mechanisms.

A Hopf and torus bifurcations in stochastic systems in mathematical population biology, in order to understanding of the interplay between deterministic mean field approximation and stochasticity, will be presented. Routes to chaos not only via the Feigenbaum period doubling but also via torus bifurcations seem more widely present in population biology, and were for example found in extended multi-strain epidemiological models on dengue fever.

Applied to infectious disease epidemiology, a model for the control of Âedes aegypti by the parasitic use of Wolbachia is investigated, proposing a control strategy to fight Dengue fever. Modeling spatial connectivity in epidemiological systems is also be presented where radiation models applied for dengue fever epidemiology in Thailand is suggested and discussed.

Two more papers deal with the use of highly sophisticated approximation techniques for assessing the basins of attraction in dynamical systems exhibiting multistability, in particular the use of flexible interpolation methods. Finally, these methods are then used in the modeling of prostate cancer, in combination with particle swarm optimization tools for the parameter assessment. 


\section{Maíra Aguiar}

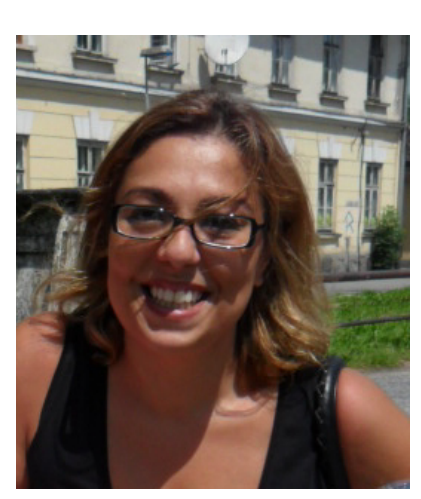

Institutional address

Lisbon University Centro de Matemática e Aplicações Fundamentais (CMAF) Mathematical Biology Group Av. Prof. Gamma Pinto, 2, 1649-003, Lisboa, Portugal. maira@ptmat.fc.ul.pt

Education/Qualifications

2008-2012 PhD in Biology, specialty in Population Biology, Lisbon University, Portugal. PhD in Life Sciences, Vrije Universiteit Amsterdam, The Netherlands.

Area of scientific activity

Epidemiology of infectious diseases; Mathematical modeling: Epidemic models development and data analysis. Presently, my research crosses the different epidemiological areas of infectious diseases by developing mathematical models which can be used by public health authorities as a tool to predict, understand the transmission of the disease and develop and evaluate the introduction of intervention strategies including vector control and vaccination.

Selected publications:

1. Maíra Aguiar, Giovanini Coelho, Filipe Rocha, Luis Mateus, José Eduardo Marques Pessanha \& Nico Stollenwerk (2015). Dengue transmission during the 2014 FIFA World Cup in Brazil. The Lancet Infectious Diseases; 7, 765-766.

2. Stollenwerk, N., Aguiar, M., Mateus, L., Rocha, F., Skwara, U., \& Ghaffari, P. (2015). Prediction and predictability in population biology: noise and chaos. Math. Model. Nat. Phenom., 10, 141-164.

3. Maíra Aguiar, Filipe Rocha, José Eduardo Marques Pessanha, Luis Mateus \& Nico Stollenwerk (2015). Carnival or football, is there a real risk for acquiring dengue fever in Brazil during holidays seasons? Nature Scientific Reports , 5, 8462; DOI:10.1038/srep0846.

4. Aguiar, M., Paul, R., Sakuntabhai, A., \& Stollenwerk, N. (2014). Are we modeling the correct data set? Minimizing false predictions for dengue fever in Thailand. Epidemiology and Infection, 142, 2447-59

5. Kooi W. B., Aguiar, M. and Stollenwerk, N. (2014). Analysis of an asymmetric two-strain dengue model. Mathematical Biosciences, 248, 128-139

6. Rocha, F., Aguiar, M., Souza, M. and Stollenwerk, N. (2013). Time scale separation and center manifold analysis describing vector borne diseases dynamics. Int. J. Computer Mathematics, 90, 2105-2125.

7. Aguiar, M., Kooi, W. B., Rocha, F., Ghaffari, P. and Stollenwerk, N. (2013). How much complexity is needed to describe the fluctuations observed in dengue hemorrhagic fever incidence data? Ecological Complexity, 16, 31-40.

8. Aguiar, M., Stollenwerk, N. and Kooi, W. B. (2012). Scaling of stochasticity in dengue hemorrhagic fever epidemics. Math. Model. Nat. Phenom., 7, 1-11

9. Stollenwerk, N., Aguiar, M., Ballesteros, S., Boto, J., Kooi, W. B., Mateus, L. (2012). Dynamic noise, chaos and parameter estimation in population biology. Interface Focus, 2, 156-169.

10. Aguiar, M., Ballesteros, S., Kooi, B. W., Stollenwerk, N. (2011). The role of seasonality and import in a minimalistic multi-strain dengue model capturing differences between primary and secondary infections: complex dynamics and its implications for data analysis. Journal of Theoretical Biology, 289, 181-196. 


\section{Roberto Cavoretto}

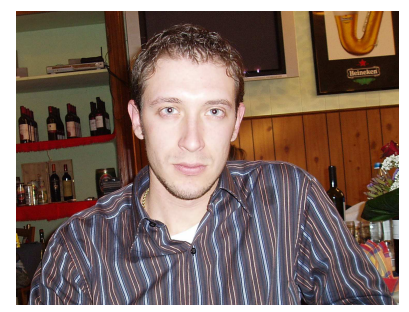

Roberto Cavoretto is a Non-Tenured Research Fellow in Numerical Analysis at the Department of Mathematics ŞGiuseppe PeanoT of the University of Torino; Ph.D. in Mathematics, University of Torino in 2010. He held a post-doc position funded by the "Istituto Nazionale di Alta Matematica"(INdAM, Rome) in 2010-2011. In 2012-13 he was invited as visiting researcher at the Illinois Institute of Technology (Chicago, USA). He was organizer and member of organizing committees of some international conferences in Numerical Analysis and Modeling, and published more than 50 referred papers. His research activity is mainly focused on topics of Applied Mathematics, such as meshfree approximation, scientific computing, mathematical modeling, system biology, and biomathematics.

\section{Nico Stollenwerk}

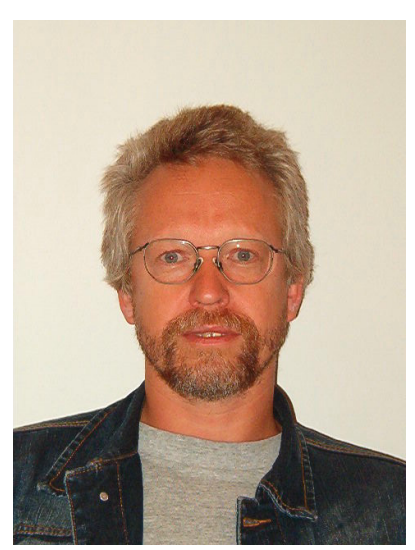

Nico Stollenwerk has graduated at University of Aachen, one of few universities with excellence status now, did his $\mathrm{PhD}$ at Clausthal University, while working as researcher at the Research Center, Juelich, the largest in Germany. He did post-doctoral studies at Cambridge University and at University of London, Royal Holloway, in the UK. Now he works as researcher at Lisbon University, leading the "Mathematical Biology group" at the Centro de Matematica e Aplicacoes Fundamentais, CMAF at Lisbon University, the center being ranked excellent in Portugal. He published besides many multidisciplinary research articles, mainly in epidemiology, ecology and neurosciences, a book with Imperial Colleg press on critical fluctuations in population biology.

\section{Ezio Venturino}

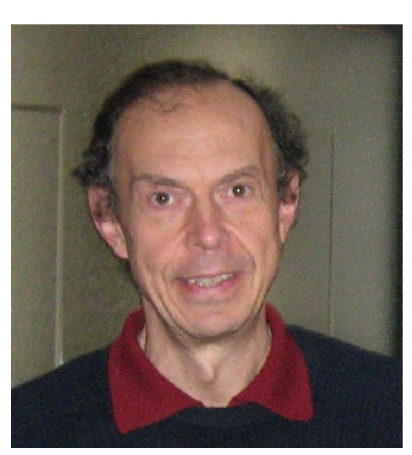

Education: Laurea in Matematica with honors (7/7/77), Università di Torino, Italy, M. Sc. (12/23/81) and Ph. D. in Applied Mathematics (12/21/84) SUNY at Stony Brook, NY, USA. Research Interests: In numerical analysis: quadrature formulae for singular integrals, methods for singular integral equations, lacunary interpolation problems. In mathematical modelling: biological population theory, ecoepidemiolgy and socioeconomic applications. Professional activities: Authored about forty research papers in numerical analysis and hundredforty in mathematical modelling. Taken part in about one hundredsixty international conferences, in some cases organizing special sessions and chairing sessions and doing organizational work. Referee for international Journals. Associate Editor of a few international Journals and for special issues of other ones. Visited for shorter or longer periods numerous international research institutions worldwide, giving also invited talks. Hosted many foreign visitors for research collaborations. Referee for several international journals. Winner of several grants of CNR (Italian National Research Council), European Training Fundation, Department of Foreign Affairs (Italy), Department of University and Research (MIUR - Italy), CRUI-DAAD Vigoni program, Postdoctoral Project WWS of the University of Torino. 